\title{
La responsabilidad del continuum educativo en el contexto del Espacio Europeo de Educación Superior
}

\author{
Moderadores: Emilio Sanz (ULL) \\ Arcadi Gual (UB, SEDEM)
}

\section{Introducción a la Mesa}

La SEDEM ha planteado esta mesa porque se acepta de forma universal que la formación de los profesionales de la medicina está inscrita en un continuum educativo (grado, Formación especializada y desarrollo profesional continuo) en el que las tres fases están relacionados y coordinadas entre si. Esta aceptación universal que acepta la formación de los médicos sujeta al continuum obedece, sin duda, a la lógica o al sentido común; sin embargo la realidad de nuestro entorno muestra como entre los tres periodos, y muy especialmente entre el grado y la Formación especializada, existe una patente desconexión tanto organizativa como conceptual. Organizativa porque estos dos periodos están sujetos a estructuras y ministerios diferentes, y conceptual porque los responsables de cada periodo no han establecido en ningún momento unas bases comunes que permitan llegar a un mismo fin.

La SEDEM ha pretendido reunir, en esta mesa, a diferentes agentes implicados para discutir la interrelación entre los dos periodos con la pretensión de obtener la visión desde diferentes perspectivas del sistema y analizar las acciones que podrían mejorar la relación entre los dos periodos.

La SEDEM, inquieta por el "gap" existente entre estos dos periodos, solicita a los invitados a la mesa redonda que antes de responder a las preguntas concretas que se les formula al final de esta introducción reflexionen sobre los tres puntos que desglosamos a continuación.

\section{1) La percepción de los actores:}

Los estudiantes: Los estudiantes tienen como prioridad el aprobar el examen MIR con la mejor nota posible lo cual es razonable. Pero ¿son conscientes de lo que pueden aprender en la universidad? ¿son conscientes de lo que pueden exigir a la Universidad?

El profesorado de Universidad: El profesorado es bueno es su materia pero ies consciente de que el objetivo del grado (la licenciatura) no es aprender su disciplina? Dicho de otro modo, el profesor universitario ¿se responsabiliza de que su disciplina este al servicio de un fin común?

El profesorado y / o los responsables de postgrado (tuto$\underline{\text { res): }}$ : Tienen la conciencia de que son los que profesionalizan a los estudiantes de medicina pero ¿tienen una visión corporativa de conjunto?

La facultad / la universidad: Se queja de que el examen MIR distorsiona el aprendizaje curricular, pero ise ha planteado algún proceso de coordinación con la Formación especializada?
Las gerencias hospitalarias y de atención primaria: Reciben a los médicos internos residentes (MIR) como profesionales para realizar asistencia, y para ello se les retribuye, pero ¿son conscientes de que fundamentalmente el MIR es un estudiante en proceso de formación?

El Ministerio de Sanidad y Consumo: ¿Se ha planteado coordinar el proceso de entrada a la Formación especializada con las facultades de medicina?

El Ministerio de Educación y Ciencia: Otorga los títulos de la especialidad, pero iasume la responsabilidad de los procesos de entrada y de salida del sistema de Formación especializada? ¿Coordina el paso de la formación de grado a la especializada?

\section{2) La realidad:}

La Formación especializada se desplazó desde las facultades de medicina al sistema nacional de salud (hospitales y atención primaria) de forma voluntaria y organizada. Esta voluntad política fue motivada por la incapacidad del sistema universitario de formar los especialistas que el sistema nacional de salud demandaba. El sistema MIR ha dado unos frutos innegables pero se ha visualizado como su mayor punto débil la falta de coordinación entre los responsables del grado y los responsables de la Formación especializada ¿No es ya el momento de superar este problema?

\section{3) Les respuestas posibles:}

Es necesario un acuerdo entre la formación que proporcionan las facultades de medicina, que se resume en las competencias de los graduados o licenciados, y los requisitos de entrada que solicita el sistema de Formación especializada. Esto conlleva, también, a que los responsables de ambas partes consensúen y coordinen el proceso de selección de entrada al sistema de Formación especializada y que actualmente conocemos como examen MIR:

Seguir como estamos. Que la universidad forme los licenciados / graduados que considere pertinentes y que el sistema de Formación especializada seleccione y ordene los estudiantes que accedan al sistema según su criterio.

Responsabilizar a la universidad. Solicitar a las universidades / facultades que en base a las competencias finales consensuadas por todos elaboren un sistema común de ordenación de los estudiantes para el acceso a la Formación especializada

Establecer un sistema de coordinación pregradoFormación especializada. Promover una estructura que facilite la comunicación entre el grado y la Formación especializada. 


\section{Javier Bayona López}

(Consejo Estatal de Estudiantes de Medicina)

\section{a) ${ }_{\dot{i}}$ Cree que la formación que se realiza en las Facultades de Medicina es la óptima para realizar la Formación especializada?}

Sin duda alguna, nuestra formación es buena pero no óptima. Actualmente los egresados de nuestra facultad están dotados de unos conocimientos teóricos generales bastante amplios, llegando a ser unos profesionales tan cualificados que no encuentran ningún obstáculo al trabajar en otro sistema sanitario de Europa o de otro lugar del mundo.

No cabe duda que el punto débil de los alumnos recién licenciados es la capacidad y manejo de habilidades practicas. Evidentemente, la irrupción del EEES deberá asegurarnos una mejora en los campos teóricos y prácticos, pero es el empeño activo de todos el que propiciara que se cumpla, porque en lugares donde ya se ha implantado de manera experimental no se han cumplido todos los objetivos.

Si deseamos acercarnos a esa meta no debemos hacer un retoque simple como se ha venido haciendo siempre en los cambios de planes de estudio, debemos implicarnos de lleno para que los alumnos de las facultades se acerquen mas a la realidad de lo que están en la actualidad. Y para ello, la figura del estudiante de medicina debe estar reconocida dentro del marco del hospital como un componente más del equipo de salud, y no un "estorbo" como sucede en la actualidad en muchos hospitales. Si alcanzásemos esa integración que en muchos países de la UE ya existe, la adquisición de habilidades será lineal con el aprendizaje teórico. Posiblemente, si esto fuese así, la duración de la residencia podría disminuir, porque ya se han adquirido competencias en el pregrado tan fuertemente arraigadas en el alumno que no es necesario volver a enseñar.

Si los ingresados de nuestras facultades han decidido dedicarse a una profesión de 6 años de carrera, 5 de especialización y que para ingresar en la universidad han demostrado una notoria media mas alta que el resto de estudiantes, es nuestro derecho el reclamar que los diferentes estamentos que están el proceso de formación (incluyendo el de alumnos) mantengan activamente su trabajo y coordinación, para que así el paso hacia la especialidad sea un transito natural.

\section{b) ¿Cree que está coordinado \\ el Grado con la Formación especializada?}

Teóricamente y desde un punto de vista ajeno a la realidad, se podría decir que en las Facultades de Medicina se nos forma excelentemente como médicos generales capaces de afrontar la realidad incluso durante el último año de carrera; que el examen MIR nos distribuye de manera ideal; y que el comienzo de la residencia no supone obstáculo alguno. Obviamente la realidad no es esa.

Si desde la facultad no se enseñan las competencias concretas y se evalúan incorrectamente, el río de nuestra formación comienza a perder agua, incrementándose más, si las vistas de futuro de algunas universidades eligen preparar a los alumnos para la prueba del examen MIR.

Si la coordinación entre MSC y MEC en temas como
EEES, RD Formativo de residentes, y otros, en los que han de compaginarse fuertemente persiste sin conexión, el continuum sigue derivando su contenido fuera.

Si seguimos sin que para la prueba del examen MIR, el MSC tenga en cuenta a todos los sectores implicados en ello (estudiantes incluidos), y una vez ya en la residencia persista la saturación de servicios, y la problemática laboral y formativa, nuestro cauce se vera extinto.

La solución es sencilla: diálogo y coordinación constantes y continuos entre todos los inmiscuidos en la tarea del continuum formativo.

\section{Jacobo Trébol López}

(Residente, Residente Servicio de Cirugía

General y del Aparato Digestivo C, H.U. La Paz;

Clínico Colaborador Docente Departamento de

Cirugía UAM)

\section{a) ${ }_{i}$ Cree que la formación que se realiza en las Facultades de Medicina es la óptima para realizar la Formación especializada?}

Para responder a esta pregunta habría que desglosar los aspectos de la formación: conocimientos, habilidades y actitudes y recordar además que la formación en las Facultades está en trámites de sufrir grandes cambios para adaptarse a las nuevas premisas educativas marcadas por el Espacio Europeo de Educación Superior.

1) Conocimientos: en la mayoría de centros de nuestro medio la formación es excelente y tiene a profesores de gran valía y reconocido prestigio para cada disciplina. Sin embargo con cierta frecuencia se aprecia que algunos de estos maestros no son realmente conscientes de que el objetivo de la carrera no es aprender su disciplina sino formar médicos generalistas. Si se pierde ese objetivo se tiende a las densas explicaciones condensadas que dificultan el aprendizaje y son poco útiles desde el punto de vista práctico. Debe tenerse claro que el licenciado debe tener dos grupos de conocimientos en cuanto a patologías se refiere:

1. Lo que debe reconocer y orientar su manejo. Para lo más relevante y prevalente (ejemplo patologías como la Hipertensión Arterial).

2. Lo que sólo debe conocer. Para aspectos menos prevalentes como por ejemplo malformaciones (cuya orientación corresponde al especialista).

Es decir, habría que conseguir que el empeño en explicar los aspectos pertenecientes al segundo grupo no dificulte el aprendizaje de los del primero (lo verdaderamente importante); muchos licenciados acaban con la sensación de tener más conocimientos acerca de malformaciones o de la fisiopatología de alguna enfermedad rara y sin embargo no saber cómo orientar el tratamiento de un simple catarro común.

En conclusión, en cuanto a los conocimientos la formación que se suministra en las facultades es de gran calidad si bien habría que concretar, en mi opinión, y remarcar mucho más que es lo más importante dentro del gran volumen de información que se imparte (posiblemente también se debería reducir dicho volumen).

2) Habilidades: es en este apartado donde probablemente se sientan los licenciados menos formados; una 
sensación muy extendida al final de la carrera es que tienen muchas carencias en la formación en habilidades; el tiempo dedicado a las prácticas en las diferentes facultades es muy heterogéneo y el aprovechamiento de las mismas lo es más todavía (generalmente se carece de programas pre-establecidos con sus objetivos y dependen mucho del clínico con el que se rote).

Tampoco se puede pretender que el licenciado tenga grandes habilidades clínicas, para eso está la Formación especializada, pero si debe tener aquellas básicas y comunes para todas las especialidades. Me parece muy acertado el enfoque que le dio a este aspecto la Conferencia Nacional de Decanos de Medicina al dividir para su Libro Blanco el saber hacer en las tres categorías de "adquirir competencia", "haber practicado bajo tutela" y "solamente haber visto". Es decir, debería haber un mínimo de habilidades básicas en las cuales deberían adquirir competencia todos los licenciados, aquel grupo de habilidades que serían comunes a todas las especialidades o aquellas maniobras que resultan útiles para cualquier médico en general.

En conclusión, para mí la formación de pregrado en habilidades debería mejorar bastante para poder asumir de forma más adecuada las grandes responsabilidades asistenciales que debe afrontar el licenciado cuando comienza la formación en su especialidad elegida.

3) Actitudes: la formación en actitudes de las diferentes facultades también es muy heterogénea; si bien cada vez son más las que intentan improntar en los alumnos una actitud crítica y analítica desde el punto de vista científico. Hoy día son muchas las facultades que imparten clases relacionadas con el análisis crítico de la evidencia, que fomentan la participación en proyectos de investigación, que organizan congresos para estudiantes y otros tipos de actividades que tienden a fomentar una actitud analítica y crítica y el trabajo basado en la evidencia científica disponible.

En conclusión diría que la formación en actitudes se ha desarrollado mucho en nuestro medio en las últimas décadas en las diferentes facultades y está en visos de desarrollarse aún más; cada vez se pone más atención en ella y esto se nota mucho al incorporarse a la residencia.

En definitiva, en mi opinión la formación suministrada por las facultades es de gran calidad para encarar e iniciar la Formación especializada si bien no se la puede catalogar de óptima especialmente por sus carencias francas en la formación en habilidades; la formación en cuanto al conocimiento debería depurarse (concretar y centrarse más en lo más frecuente) y la de actitudes todavía debe mejorar y homogenizarse entre los diferentes alumnos y los distintos centros formativos.

\section{b) ¿Cree que está coordinado el Grado con la Formación especializada?}

En mi opinión existe bastante descoordinación entre ambas etapas formativas. Entre otros porque dependen de ministerios diferentes.

\section{Coordinación grado-acceso a la Formación} especializada:

El tránsito entre ambas etapas está regulado por la realización de una prueba (el examen MIR) que es una evaluación puramente teórica (tipo test, consta de 250 preguntas). En ella se valoran conocimientos de las diferentes especialidades seleccionados de forma azarosa; da la sensación de que no se valora ni lo más importante ni lo más frecuente; afortunadamente en los últimos años se ha producido un giro en la modalidad de las preguntas y ya no hay tantas puramente teóricas sino que cada vez son más las formuladas en formato caso clínico, lo que se acerca un poco más a la realidad de nuestra actividad. Es decir, el examen está poco a poco girando hacia un sentido más práctico.

Hay varios aspectos perniciosos en la formulación actual del examen. Al representar tanto respecto a la valoración final para la solicitud de una plaza de la Formación especializada termina mediatizando a los alumnos, especialmente en los últimos años de la carrera. Llega a haber momentos en los que están más preocupados de iniciar y cumplimentar la formación específica para el examen (frecuentemente dependiente de academias privadas) que de completar su currículo de pregrado o de acudir a las prácticas para mejorar sus, deficientes en muchos casos, habilidades clínicas.

No parece adecuado que para mejorar los resultados obtenidos en ese examen gran parte de los alumnos deban recurrir a realizar preparaciones específicas en organizaciones privadas; esto es un marcador de la escasa coordinación entre el Grado y el acceso a la Formación especializada. Las facultades deberían aportar lo suficiente para superar esa prueba, y de hecho lo hacen e incluso aportan mucho más, pero también deberían preocuparse de aportar ese grano extra de formación concreta que aportan las academias y que tan útil resulta (lo demuestra el uso masivo histórico por los alumnos): entrenamiento en técnicas de test, selección de la información más preguntada, entrenamiento en preguntas test tipo caso clínico, etc.

Parece poco razonable que se pongan por un lado seis años de exámenes y estudio y por el otro un año (o dos) y un solo examen y que lo primero represente el 25 y lo segundo el $75 \%$ de la valoración final para el acceso a la Formación especializada. Para solventarlo habría que, entre otras cosas, homogenizar más la formación de Pregrado entre los distintos centros y establecer un sistema de mínimos (como el marcado por el Libro Blanco) y probablemente habría que dar más importancia los resultados obtenidos por el esfuerzo continuado durante seis exigentes años.

Además el planteamiento del examen no es probablemente el más adecuado dado que las diferentes preguntas las ponen especialistas y, en opinión de muchos alumnos y profesores de la facultad, son frecuentemente muy concretas y demasiado especializadas que probablemente exceden ese objetivo de "valorar médicos generalistas" si bien ha cumplido hasta el momento una función clasificadora para el acceso a la Formación especializada.

\section{Coordinación con el resto de la Formación} especializada:

Como ya hemos reseñado previamente el aspecto en el que más deficitaria es esta coordinación es la formación en habilidades clínicas relacionadas con la especialidad. 
Esta descoordinación tiene que ser aún mayor en aquellos centros no adscritos a universidades que en general tienen menos tradición docente.

Para mejorar esta coordinación hay muchas actividades que podrían ayudar a aumentar la comunicación entre ambos como:

Reuniones entre representantes de tutores y de profesores de la especialidad concreta.

Reuniones periódicas de los altos cargos: representantes de ambos Ministerios para mejorar la coordinación.

Reuniones entre esos altos cargos y la base del sistema (los MIR) a través de sus representantes.

Implicar en la docencia de alumnos de Pregrado, especialmente en la parte práctica, a los MIR; que tienen más tiempo y mucha mayor proximidad y complicidad con los alumnos y así podrán probablemente mejorar la deficitaria formación en habilidades. Es muy probable que así se consiga mejorar la coordinación entre los sistemas de formación de ambos períodos. En la UAM se ha aplicado un sistema de este tipo en el contexto de un proyecto denominado "El residente como maestro" que ha utilizado como plataforma un cursillo conocido como "Iniciación a la Práctica Médico-Quirúrgica". En él se ha insistido en dos aspectos: implicar a los residentes en la docencia y la docencia en habilidades (llegando incluso a evaluarlas en un examen tipo ECOE). Los resultados de sus evaluaciones y de las encuestas de satisfacción demuestran el éxito del programa y una mejoría en la formación de habilidades de sus alumnos lo que podría ayudar a encarar mejor la Formación epecializada.

\section{c) ${ }_{\grave{i}}$ Cree que la Formación especializada es la adecuada para ejercer la especialidad?}

La Formación especializada es un sistema en mi opinión muy válido siempre y cuando funcionen todos los implicados en el mismo. Si bien en cuanto falla alguno puede incurrirse en graves defectos de formación que pueden terminar incluso originando acontecimientos de mala praxis médica.

\section{La formación del MIR:}

El sistema se sustenta demasiado en la responsabilidad que adquiere en su formación el MIR; MIR que en muchas ocasiones sufre una gran carga asistencial que dificulta disponer de tiempo para el estudio: número de guardias, no libranza de las mismas, diversificación de su actividad, actividades vespertinas (cirugías o consultas de tarde, etc que frecuentemente no aportan un gran aprovechamiento). Es decir, el MIR frecuentemente tiene una carga laboral excesiva que puede comprometer su aprendizaje y que además está en general poco reconocida.

La adquisición de habilidades no siempre es homogénea dado que no siempre se dan las condiciones para que todos puedan adquirirlas (especialmente en los centros de bajo número de camas); en estos casos deberían establecerse sistemas de mínimos (de pacientes manejados con determinada patología, de un determinado procedimiento quirúrgico, etc) y sistemas de rotación para adquirir aquellas habilidades que no puedan adquirir en su centro (por ejemplo en especialidades quirúrgicas sin transplantes tener establecida una rotación externa).
Debe destinarse tiempo a la formación en actitudes, es decir la formación en metodología científica y en investigación. Algunos nuevos programas de especialidades incluyen ya claros epígrafes al principio de la residencia destinados a cursos de iniciación a la metodología científica y el análisis crítico de la ingente información médi$\mathrm{ca}^{10}$. Debe asumirse como natural que el residente adquiera estos conceptos y participe en la investigación, y que eso no tiene porque ser patrimonio exclusivo de su tiempo libre sino que forma parte de su formación integral. Este tipo de formación y participación en la investigación no se da en todos los centros y en los que se tiene la oportunidad la mayoría de las veces es a expensas del tiempo libre del residente.

\section{Análisis de la figura del Tutor:}

No hay unos criterios de selección claros ni se le han asignado los mínimos recursos para su labor: formación específica, tiempo y reconocimiento.

Esta responsabilidad suele recaer en alguno de los especialistas del servicio, con un nivel de conocimientos de la especialidad medio-alto y voluntarioso, que se enfrenta a esa labor cargado únicamente, y en el mejor de los casos, con su buena voluntad y motivación. Otras veces, cuando no se consigue encontrar a nadie motivado, se nombra directamente a algún miembro del servicio, aunque no tenga vocación docente, o termina asumiendo este papel el jefe del servicio.

Hasta ahora, este modelo basado en la buena voluntad $\mathrm{y}$ paternalista ha funcionado de forma variable. Sin embargo, los nuevos retos de formación a los que se enfrentan las diferentes especialidades lo ponen en tela de juicio.

Por un lado, la sociedad exige un especialista que actúe de acuerdo con sólidos principios científicos y en permanente actualización; bien formado académicamente y bien entrenado en habilidades clínicas. Por otra parte la tendencia es hacia una reducción del número de horas laborables, que en el caso de los residentes equivalen a formación y adiestramiento. En definitiva nos encontramos con una formación cada vez más exigente y sobre la que se ciñe la amenaza de una disminución del período de aprendizaje lo que requiere un replanteamiento de cómo llevarla a cabo.

Se va a precisar otro modelo de tutor, uno que cumpla unos criterios de preparación y selección, que dedique el tiempo necesario, al que se pueda exigir el cumplimiento de sus funciones, que sea evaluado y que reciba una compensación por esta labor. En resumen: perfil, acreditación y reconocimiento de su exigente actividad.

El perfil no sólo se refiere a que el Tutor debe ser un buen especialista sino que también debe tener una formación específica como docente y pedagogo, capacidad organizativa y de manejo de grupos humanos y motivación para la docencia y la investigación. Diferentes entes están trabajando ya en la parte del perfil. Por ejemplo la Organización Médica Colegial ha publicado recientemente el "Manual para el Tutor MIR" en un esfuerzo para mejorar su formación específica, concretar sus responsabilidades, etc; y algunas organizaciones profesionales, como la Asociación Española de Cirujanos, han organizado ya cursos y congresos para tutores. Por supuesto en 
este aspecto también se hace notar el trabajo de la SEDEM (organizando por ejemplo los "Encuentros de Tutores"-quinta edición en 2007).

En cuanto a la acreditación sería deseable que se acreditara su perfil y que fuese sometido a evaluaciones de sus resultados como docente.

Es más complejo el componente del reconocimiento que debería ser por una parte curricular (y para los baremos de promoción profesional) y por otro lado económico. Este último elemento puede ser polémico en tanto en cuanto se puede aducir que todos los elementos de una unidad docente participan en la docencia (de hecho están obligados a ello al pertenecer a una unidad con docencia acreditada). Sin embargo sobre el tutor recaen más responsabilidades como la de organizar y garantizar el plan formativo individual de cada residente; además el tener que dedicar tiempo a estas actividades no suele suponer un descenso de su carga laboral habitual por lo cual suelen tener que cumplir estas funciones frecuentemente fuera de su horario laboral.

En resumen el sistema es bastante adecuado si bien hay algunas lagunas importantes que habría que subsanar. En primer lugar esforzarse porque todas sus piezas funcionen engranadas a la perfección. En la vertiente del receptor de la enseñanza (el residente) en cuanto a la adquisición de conocimientos habría que conseguir que el volumen de trabajo no interfiera con la misma; en las habilidades habría que homogenizar y sistematizar la adquisición de las mismas estableciendo un sistema de mínimos y permitiendo las rotaciones externas para completar las exigidas por el plan de formación y en las actitudes habría que insistir en que la formación en metodología e investigación pertenece al global de la residencia sin estar reñida con la actividad asistencial. Con una buena organización de los servicios los residentes pueden perfectamente realizar investigación, participar en cursos, ir a congresos, etc y esto debe asumirse como parte de su actividad normal en cualquier centro (si no lo hay en el propio usar colaboraciones con otros centros). En cuanto a los responsables de esa formación (los tutores) podría resumirse en tres conceptos: perfil, acreditación y reconocimiento.

\section{d) ¿Debería evaluarse formalmente la Formación especializada al término de la misma?}

Probablemente debería evaluarse pero de una forma positiva. Lo ideal sería llevar a cabo un examen de mínimos para garantizar un bagaje de conocimientos relacionado con la especialidad; positivo porque permitiría detectar ciertos déficits e intentar ponerles solución antes de la definitiva incorporación al mundo laboral de la especialidad ya sin la supervisión de los demás. Por ello creo que debería realizarse un tiempo antes del final de la residencia para así permitir al tutor trazar un plan "de emergencia" para solucionar posibles defectos formativos.

Lo más sencillo sería llevar a cabo una evaluación de conocimientos, la de las habilidades y actitudes ya se ha ido llevando de forma continuada durante la convivencia laboral del residente con el tutor y otros elementos de la unidad docente. Si bien no estaría de más hacer una revisión detallada de lo realizado hasta el inicio del último año y trazar un plan intensivo el último año para cumplir los objetivos de ese año y aquellos déficits que se hayan detectado en los años previos.

Esta evaluación debería ser además del tipo "board" europeo o incluso el estadounidense. De esta forma además se conseguiría tener la homologación del título de la especialidad con otros sistemas sanitarios, sobre todo en estos tiempos en los que cada vez son más las demandas de médicos españoles desde otros sistemas al tener ellos un déficit importante de profesionales que tratan de cubrir con médicos extranjeros, siendo los españoles uno de los grupos preferidos en dicha selección.

\section{José $\mathbf{M}^{\mathrm{a}}$ Peinado Herreros}

(Universidad de Granada, Conferencia nacional de Decanos)

\section{a) ¿Cree que está coordinado el grado y la Formación especializada?}

Creo que hay mucho por mejorar en los aspectos de coordinación, no solo entre grado y Formación especializada, sino que también es absolutamente necesaria la coordinación dentro del grado y dentro de la propia Formación especializada.

Aún partiendo de que los estudios de medicina son de los mas definidos en cuanto a contenidos, con directiva europea, un cuerpo de doctrina establecido a lo largo de siglos y una actividad profesional altamente regulada, uno de los principales problemas en el grado deriva de que nuestro país no ha definido con suficientemente los objetivos y competencias de la titulación. Esto dificulta el realizar un constructo sobre el producto de las facultades de medicina. En consecuencia es cada facultad de medicina, en base a sus recursos, filosofía docente, tradición, etc.. quién interpreta lo que hasta ahora se ha denominado troncalidad, definida en el BOE de directrices de la titulación.

No existe, en general, una coordinación adecuada entre los contenidos de las diferentes materias dentro de cada facultad. Se desdibuja desde el principio la visión integral de conjunto, y se produce una excesiva parcelación relacionada con asignaturas y departamentos. La actual normativa universitaria concede a los departamentos, en detrimento de los centros, una parte muy sustancial de las decisiones que tienen que ver con la definición de los contenidos de las materias y la planificación de los recursos humanos. Los departamentos no suelen interpretar la carrera como un conjunto, bien al contrario, sus decisiones suelen estar mediadas por intereses personales, promoción de su profesorado u otros elementos. Esto impide, además, una adecuada política de recursos humanos o infraestructuras. No existe "autoridad" a la hora de definir o coordinar programas, y menos aún a la hora de concretarlos en la docencia de un profesor, que en general se considera ultimo interprete, ante una libertad de cátedra mal entendida.

Si no existe una definición de competencias, particularmente de los conocimientos, mucho menos de las habilidades clínicas o de comunicación o de actitudes. Los alumnos acceden a los centros asistenciales sin una programación definida de su papel en los mismos y en los procesos que en ellos se desarrollan. Mucho menos valor 
se le da aún a la evaluación de habilidades y actitudes, centrándonos todavía, en la evaluación de conocimientos. Además estos, mucho menos las habilidades, no siempre tienen una relación directa con la práctica clínica habitual. El saber enciclopédico sigue jugando un papel prevalente en nuestro sistema, pero es difícil modificarlo dadas las características del examen MIR, centrado exclusivamente en la valoración enciclopédica y rebuscada de conocimientos. La proliferación de academias MIR es buena prueba de ello.

Respecto a la coordinación con la Formación especializada, aparecen problemas similares. En un momento en que se está produciendo un intenso debate sobre las diferentes especialidades médicas y su organización por troncos, se ignora de forma absoluta la formación de grado. No es infrecuente encontrar en los programas de la especialidad cursos, por ejemplo, sobre estadística, informática, u toros aspectos de formación básica realizados en el grado. La mayoría de las especialidades siguen defendiendo sus respectivas peculiaridades, y aparecen dificultades en el establecimiento de años básicos comunes. Se asume con gran naturalidad, que se parte casi de cero en habilidades clínicas o de comunicación, aunque luego la realidad de los R1, asume una formación que se ha predefinido como "inexistente". En cualquier caso todo este debate se realiza sin que el Ministerio de Sanidad se siente con el de Educación, y menos con las facultades de medicina, a fin de definir las competencias que deben tenerse al comienzo de la residencia.

Esta situación se agrava en un entorno de competencias en salud y educación transferido a las CCAA, y en el que el establecimiento de conciertos entre las instituciones sanitarias y las universidades se basa en una normativa de 1986, completamente desfasada, y que dificulta o casi impide la carrera académica de los médicos. Ninguna de las modificaciones normativas a la que se ha sometido a la universidad en los últimos años ha considerado la situación de las facultades de medicina. Los profesores en materias básicas cada vez poseen una menor formación médica, y los profesores de materias clínicas, cada vez tiene mayores dificultades para compatibilizar su actividad asistencial con la docente y mucho mas con la investigadora. Tampoco se valora suficientemente la docencia. Esto ha devenido en una gran dificultad para encontrar profesorado bien formado en las facultades de medicina.

La ocasión que se presenta en los próximos años es única. La reforma del grado, del examen de acceso a la especialidad y de la Formación especializada deberían discurrir de la mano y coordinadamente. Las facultades de medicina tenemos la firme voluntad de prestar nuestra colaboración en dicho proceso. No obstante la persistente ignorancia por parte de la administración del papel de las facultades en dicho proceso, junto a general desconocimiento de la realidad docente en nuestros centros de las autoridades universitarias y políticas, me hace ser escéptico.

\section{b) ¿̨Tiene que estar la Universidad involucrada en el acceso a la Formación especializada?}

Con rotundidad sí, y así lo manifestó al Ministerio de Sanidad, la Conferencia Nacional de Decanos hace más de
5 años. El acceso a la Formación especializada supone el paso obligado para cualquier titulado en medicina, y la continuación imprescindible de su formación. Parece por tanto lógico que el sistema de acceso se fundamente en la formación obtenida en las facultades de medicina. Por el contrario, a lo largo de los años el proceso se ha invertido. Han sido las facultades de medicina las que se han adaptado al formato del examen, tanto en el propio proceso de formación como en el de evaluación. A mi juicio debería de reflexionarse en torno a los siguientes aspectos:

La confección de la prueba MIR debería considerar los contenidos y el peso de las distintas materias reconocidas en las directrices generales y en los planes de estudio de la licenciatura en medicina, con particular relevancia de las áreas clínicas. La prueba debería incluir la evaluación de las habilidades clínicas y las actitudes previamente definidas, a través de herramientas reconocidas a nivel internacional. En definitiva, el sistema de acceso debe basarse en la exigencia de competencias previa, clara y específicamente definidas.

La participación de las facultades en el acceso a la Formación especializada, debe entenderse básicamente en tanto definición de objetivos, contenidos y competencias, previamente acordadas con los responsables de educación y sanidad. En consecuencia, las preguntas deberían originarse en el propio desarrollo curricular de todas las facultades de medicina del país. Sin embargo no necesariamente deben evaluar los mismos implicados en el proceso de enseñanza-aprendizaje, o lo que es lo mismo que el examen deban realizarlo las facultades. Podría ser realizado por equipos evaluadores externos a los centros, entre cuyos miembros hubiera profesorado de las facultades, conectados y con conocimiento de la dinámica que se siguen en todas las facultades de medicina.

Aún cuando se asume que para ser discriminatoria la prueba debe introducir cuestiones con diferentes grados de dificultad, debería de alejarse del detalle irrelevante.

Los resultados totales y particulares de la prueba MIR, deberían ser conocidos por todas las facultades de medicina, a fin de posibilitar el reconocimiento de sus carencias y el establecimiento de mecanismos correctores. La ausencia de información objetiva, ante las aparentes y reiteradas diferencias obtenidas en la prueba por las diferentes facultades (solo se publican los 100 primeros alumnos y sus facultades de origen), no se percibe como limitaciones de unos centros respecto a otros, sino mas bien en términos de agravios y ventajas.

Además, creo muy importante la puesta en marcha de mecanismos de evaluación y acreditación de los centros y titulaciones. Esto debe ser un requisito imprescindible, y previo, a cualquier proceso de evaluación de los alumnos, y por tanto del examen MIR. Al igual que las unidades docentes para la Formación especializada necesitan acreditación y se define, en base a su infraestructura, el número de plazas de formación, las facultades de medicina deberían cumplir una serie de requisitos mínimos imprescindibles que aseguren la calidad de formación de un número de estudiantes adecuado a sus recursos. Dichas acreditaciones deben ser adjudicadas por evaluadores independientes, ajenos a la institución. 
Otro tema que no puedo soslayar en la necesaria participación de las facultades de medicina en el acceso a la Formación especializada es la homologación de títulos extranjeros. Nada que objetar a la homologación automática de títulos de la UE, mas allá de las imprescindibles pruebas de certificación de idioma a un nivel adecuado para una correcta comunicación médico-paciente.

Respecto a la homologación de títulos no UE, y que de facto supone el acceso a la prueba de Formación especializada, debería haber una implicación directa de las facultades de medicina, con un comité único de carácter nacional que homologase la competencia médica, alejándonos del mero tramite administrativo. En nuestro país se homologan cada año unos 3000 títulos de licenciado en medicina, fundamentalmente de países iberoamericanos, lo que iguala la producción de 20 de las 28 facultades de medicina españolas. No parece razonable que acceder a las facultades de medicina españolas y obtener el título, se realice en unas condiciones de gran exigencia, y homologar el título se limite, en más del 99\% de los casos, a un trámite burocrático, en el que no se demuestra en ningún caso la competencia clínica.

\section{Matilde Ezquerra Lezcano}

(Asociación de Redes de Docencia y Asesoras)

\section{¿Los recursos humanos de la formación especializada, los tutores, tiene la formación adecuada, los recursos necesarios y el reconocimiento suficientes para desarrollar su función docente?}

La formación especializada por el sistema mir tiene aproximadamente 40 años de antigüedad en España, coincidió con la creación de los nuevos hospitales de la seguridad social. Surgió de la necesidad de un tipo de profesional con una formación más actualizada, dispuesto a trabajar en equipo y a tener dedicación exclusiva, fue iniciativa de un grupo de profesionales inspirados en modelos anglosajones. Podemos destacar dos características fundamentales que han perdurado desde su inicio: el aprendizaje durante el trabajo y la elevada participación de los profesionales asistenciales en el proceso. Estas características fueron y siguen siendo las diferencias más importantes respecto a la formación de pregrado, han supuesto aspectos positivos y otros no tanto, desde un punto de vista metodológico la formación en servicio tiene un potencial de aprendizaje mucho más alto, teniendo en cuenta que es una formación de adultos. El Dr. Albert Oriol citaba en alguna ocasión que "el conocimiento útil no se adquiere tanto cuando se aprende como cuando se utiliza". La otra característica a destacar es la vinculación tan estrecha entre la formación y el sistema asistencial esto ha supuesto una actualización constate de los conocimientos, habilidades, actitudes y valores transmitidos por los profesionales a los futuros especialistas. También podemos citar aspectos no tan positivos de esta formación como sería en muchas ocasiones la improvisación, el excesivo peso de un aprendizaje por imitación, la potenciación de la estandarización y adquisición del "rol" por encima de la reflexión, y ocasionalmente una excesiva rapidez en la incorporación en el mundo profesional sin poder desarrollar plenamente la etapa de la formación especializada.

No se dispone de un registro centralizado del numero de tutores a nivel del Ministerio de Sanidad para tener una idea aproximada de los recursos humanos podemos hacer una extrapolación teniendo en cuenta el numero de plazas de la última convocatoria (alrededor de 6000), la duración de la mayoría de especialidades (4 años) y una asignación de 2-4 residentes a cada tutor esto supondría que existen entre 6000 y 12000 tutores en el sistema de formación especializada. También podemos tener una idea de la estructura de formación si tenemos en cuenta el numero de centros hospitalarios acreditados (250) y unidades de medicina de familia y comunitaria (93). Cada uno de estos centros o unidad docente posee una comisión de docencia o asesora para su funcionamiento. Estas cifras nos dan idea de que la formación especializada es muy participativa sobre todo si lo comparamos con las 29 facultades de medicina del territorio que pueden tener no más de 80 centros universitarios. Esta alta participación ha vinculado la formación especializada al sistema asistencial y al desarrollo profesional mediante la conexión que tienen los profesionales con las sociedades científicas, tenemos algunos ejemplos de sociedades científicas que tienen secciones de residentes, que realizan formación específica para estos o que incluyen en las secciones de docencia un apartado importante destinado a la docencia de la formación especializada. No ocurre así con la Universidad donde los puentes de relación con las estructuras responsables de la formación especializada (jefes de estudio y tutores) son prácticamente inexistentes.

$\mathrm{Al}$ intentar describir la estructura organizativa de la formación especializada se pone de manifiesto su amplitud como decía anteriormente pero también su debilidad, es decir, todavía estamos en una fase donde la formación especializada es un "añadido" para la mayoría de los responsables asistenciales, no esta planteado como una línea de producto independiente en términos de gestión, de la atención de los pacientes. Esto comporta un desarrollo con insuficiente entidad de los recursos destinados a la docencia, de la valoración de los profesionales dedicados a esta tarea y ocasionalmente tensiones entre las necesidades asistenciales y docentes. En los últimos años podemos ver alguna iniciativa de cambio planteada por las administraciones autonómicas como por ejemplo la creación de un contrato específico para la docencia especializada con cada uno de los centros.

El proceso de transferencias a las Comunidades Autónomas (CCAA) de las competencias en materia sanitaria se ha completado en los últimos años. Se ha transferido prácticamente toda la capacidad de decisión en el ámbito asistencial y es necesario profundizar en la descentralización de la formación especializada. Esta necesidad se justifica en las diferencias que se observan entre las distintas CCAA, entre sus problemas y prioridades, también en el terreno docente. En el marco de lo que dispone la Ley de Ordenación de las Profesiones Sanitarias (LOPS), se esta trabajando en diferentes aspectos de regulación de la formación especializada, de desarrollo de 
la troncalidad etc...la aplicación de estas reformas tendrá necesariamente que tener una orientación autonómica para permitir una mejor adaptación al nuevo contexto de la política sanitaria estatal.

Las CCAA deben desarrollar y consolidar su estructura docente en formación especializada. En este sentido desde hace unos años ha supuesto un paso importante la constitución en muchas CCAA de redes que agrupan las diferentes comisiones de docencia y asesoras como por ejemplo la XARXA de Cataluña, REDECA de Andalucía, Redfesma de la Comunidad de Madrid, REDOVA de la Comunidad Valenciana o REDEGA de la Comunidad de Galicia. El objetivo de estas redes es coordinar a los diferentes centros docentes y realizar propuestas de mejora del proceso de formación. De la misma forma un poco posterior se constituyó AREDA (Asociación de Redes de Docencia y Asesoras) en octubre de 2004 con el objetivo de agrupar y coordinar las actuaciones de las redes autonómicas. Estas asociaciones están facilitando el intercambio entre los docentes y organizando actividades de formación específicas sobre todo de aspectos pedagógicos. También determinadas organizaciones vinculadas a la administración como el Instituto de Estudios de la Salud (IES) o la Agencia Laín Entralgo o La Fundación Iavante están jugando un papel muy activo en relación a la formación de los docentes y en definitiva contribuyendo con esto a la consolidación de esta estructura.

En relación al reconocimiento de los docentes, el nivel de desarrollo ha sido muy heterogéneo tanto desde el punto de vista de las diferentes especialidades como desde el punto de vista de las diferentes CCAA. Por poner un ejemplo en Cataluña se iniciaron las primeras experiencias en la especialidad de Medicina Familiar y Comunitaria en el año 1991 para finalmente disponer de un sistema de acreditación externa de tutores de formación especializada en el año 2006.

La necesaria consolidación de esta estructura de formación especializada reforzando sus recursos humanos (jefes de estudio y tutores) como piezas claves es un paso imprescindible de identificación como grupo, de fijación de objetivos, de evaluación etc. que debería permitir iniciar una relación y colaboración más estrecha con las Universidades y los responsables de la formación de pregrado.

\section{¿Las nuevas unidades docentes dan respuesta a las necesidades de la formación especializada?}

Se esta trabajando en un nuevo concepto de unidad docente definido como "el conjunto de recursos personales y materiales, pertenecientes a dispositivos asistenciales, docentes de investigación o de cualquier otro carácter que, con independencia de su titularidad, se consideren necesarios para impartir formación reglada en especialidades en ciencias de la salud por el sistema de residencia, de acuerdo con lo establecido en los programas oficiales de las distintas especialidades" (proyecto de real decreto, julio 2007). Esta nueva definición facilita la creación de unidades docentes dotadas de diferentes recursos independientemente de la Institución a la que pertenezcan haciendo énfasis en las necesidades del programa de formación esto va a permitir una adaptación mayor a la realidad del sistema sanitario y dar más peso a la unidad docente en relación al centro. Podemos considerar como antecedente las unidades docentes de Medicina Familiar y Comunitaria, unidades docentes de una sola especialidad, esta característica junto a la dotación de una infraestructura propia menos vinculada a un único centro y por tanto más alejada de la gestión asistencial ha permitido un mayor trabajo de coordinación entre los responsables y consecuentemente un mayor desarrollo de los programas de formación.

También se introducen unas nuevas unidades docentes de carácter multiprofesional en las que se formaran diferentes especialistas: Unidades docentes de Salud Mental (psiquiatría, psicología clínica, enfermería en salud mental), Pediatría (pediatras y enfermería pediátrica), Atención Familiar y Comunitaria (medicina familiar y comunitaria y enfermería familiar y comunitaria), Salud Laboral ( medicina del trabajo y enfermería del trabajo), Geriatría (geriatras y enfermeros geriatras) y Obstetricia y Ginecología (obstetricia y ginecología y matronas). También prevé la existencia de unidades docentes multiprofesionales de carácter troncal. Estas unidades tendrán se adscribirán a una comisión de docencia o tendrán una propia. El residente aprende durante la etapa de la formación especializada el rol que desarrollará posteriormente como profesional, por este motivo establecer una única unidad docente que gestione la formación de estos profesionales puede ser una buena oportunidad para facilitar el trabajo en equipo, tan necesario para la buena calidad asistencial.

\section{Alfonso Moreno González}

(Consejo Nacional de Especialidades, Universidad Complutense de Madrid)

\section{a) ¿Cree usted que está coordinada la formación de grado y la Formación especializada?}

Si tuviera que responder con una sola palabra diría NO, no hay coordinación entre la formación de Grado y la Especializada. Ello es así y hay algunos hechos que lo apoyan; en mi opinión, en la elaboración de los contenidos de los programas de las diferentes asignaturas del Grado se tiene muy poco en cuenta que el médico que debe salir de la facultad debe ser un médico con una visión general y amplia de la Medicina y no un miniespecialista en distintas especialidades. Todavía, a día de hoy, hay Facultades en donde el alumno no realiza ninguna práctica en consultas de Medicina General o Medicina Familiar y Comunicaría, pero sí puede haber participado en una práctica sobre transplante hepático. Creo que lo que pueden representar estas situaciones deben de corregirse para que desde la facultades se formen médicos generales y básicos capaces luego, durante la Formación especializada, de asumir la formación más específica en cada una de las especialidades.

Otro hecho que puede corroborar esa falta de coordinación es que, formalmente, nunca ha habido un foro de discusión o de intercambio de propuestas entre la Conferencia 
de Decanos de facultades de Medicina y el Consejo Nacional de Especialidades en Ciencias de la Salud, que son los dos organismos que, en su ámbito de competencias, pueden y deben hacer posible esa coordinación.

Pero por otro lado, podría entenderse que un cierto grado de coordinación existe ya que en la Comisiones Nacionales de cada una de las especialidades hay dos vocales que propone el Ministerio de Educación y que deben ser profesores universitarios, por lo que también tienen actividad en los estudios de Grado.

Igualmente, en la elaboración del temario del cuestionario de la prueba de acceso a la Formación especializada participan, a título individual, distintos profesores de Facultades de Medicina.

Aún así, es evidente la necesidad de una mayor implicación, en la coordinación, entre los entes responsables de la formación de grado y de la Formación especializada.

\section{b) ¿Cuáles serian los mecanismos para favorecer/mejorar esa relación?}

Es necesario potenciar la coordinación que debe hacerse de forma paulatina y progresiva para que no existan "fracturas" entre ambos periodos formativos.

Un buen inicio de ello podría ser una mayor participa- ción de la facultades en la elaboración de la nueva prueba de acceso que, como señala la LOPS, debe de incluir además de conocimientos teóricos y prácticos y habilidades clínicas y comunicativas.

También la integración más efectiva del alumnado en los Servicios Clínicos acreditados para la Formación especializada, con la implicación activa de los residentes en las prácticas clínicas de los alumnos podría ser otra forma de favorecer la coordinación.

Igualmente es imprescindible en la formación del alumno, el que todo el "staff" del Servicio tenga la consideración docente para el Grado, pues se da el hecho curioso de que la plantilla completa de un Servicio con acreditación docente participa en la Formación especializada del residente y así se reconoce normativamente, pero no puede hacerlo en la formación del alumno de Grado ya que no todos los médicos de la plantilla son así reconocidos por la Universidad.

Creo que puede haber más mecanismos que favorezcan la coordinación, que deben ser puestos en marcha de la forma más adecuada ya que, como indica la LOPS, todas las estructuras sanitarias estarán a disposición de ser utilizadas para la investigación y la docencia de los profesionales sanitarios. 